\title{
PERTINENCIA DE LA FORMACIÓN TÉCNICA DE NIVEL SUPERIOR EN RELACIÓN CON LA ESTRATEGIA NACIONAL DE INNOVACIÓN PARA LA COMPETITIVIDAD
}

\section{Pablo Carrasco, Juan Pablo Venables ${ }^{1}$}

\section{RESUMEN}

El desarrollo de una estrategia nacional de innovación para la competitividad sienta las bases, por primera vez en Chile, del horizonte de desarrollo por el cual nuestro país espera transitar en los próximos años. Sin embargo, para que esta estrategia pueda alcanzar los objetivos planteados, requiere que previamente sean abordadas las brechas detectadas para su implementación. En materia de capital humano, este estudio revisa la oferta y demanda por formación técnica de nivel superior y analiza la pertinencia de su comportamiento según los clusters de desarrollo productivo, constatando la falta de articulación entre ambos sectores e identificando algunos desafíos.

Palabras clave: cluster, innovación, formación técnica, oferta, demanda, pertinencia.

\section{RELEVANCE OF TECHNICAL HIGHER EDUCATION IN RELATION TO A NATIONAL INNOVATION STRATEGY FOR COMPETITIVENESS}

\section{ABSTRACT}

For fist time in Chile, the creation of a national innovation strategy for competitiveness lays the foundations for the development path our country should follow in the coming years. For its implementation, however, it is necessary to address some gaps previously identified. In terms of human capital, this article reviews the supply and demand for technical higher education and discusses its relevance in relation to productive development clusters noting the lack of coordination between both sectors and identifying some challenges.

Key words: cluster, innovation, higher technical education, supply, demand, relevance.

1 Consejo Nacional de Educación, Chile. Contacto: pcarrasco@cned.cl 


\section{PERTINENCIA DE LA FORMACIÓN TÉCNICA DE NIVEL SUPERIOR EN RELACIÓN CON LA ESTRATEGIA NACIONAL DE INNOVACIÓN PARA LA COMPETITIVIDAD}

\section{Introducción}

Durante los últimos 17 años, Chile ha avanzado exitosamente en ampliar la cobertura de educación superior, pasando de poco más de 250 mil estudiantes a más de 820 mil en 2009 (MINEDUC, 2009), lo que representa un crecimiento superior al 300\%. La masificación de este nivel educacional ha permitido la incorporación de grupos sociales hasta hace poco excluidos del sistema, los que han generado una demanda importante por vacantes, impulsando un significativo crecimiento de la oferta de programas de formación superior.

Sin embargo, persisten otros problemas que afectan a este nivel educacional, referidos a la calidad de la oferta de formación y su pertinencia, que son hoy objeto de debate. Por parte del sector público, estos asuntos han sido abordados mediante el surgimiento del Sistema Nacional de Aseguramiento de la Calidad, implementado preferentemente a través de procesos de acreditación (institucional, de pre y posgrado), el que ha generado consensos respecto de la necesidad de examinar periódicamente la oferta de formación superior. En este contexto, alcanzar una adecuada articulación entre esta oferta de formación y las demandas del sector productivo, favoreciendo la efectiva incorporación de los egresados en el mercado laboral, adquiere vital importancia.

Si bien ésta es una preocupación común para el conjunto del sistema, representa un especial desafío para la formación técnica de nivel superior, toda vez que Chile presenta un déficit importante de técnicos preparados para enfrentar los desafíos productivos del país y la oferta actual de carreras de este tipo carece de evidencia respecto de su pertinencia en relación con las demandas del mercado laboral. 
Sin embargo, en la actualidad, el país cuenta con las herramientas para abordar esta situación.

Efectivamente, fruto del trabajo desarrollado desde 2005 por el Consejo Nacional de Innovación para la Competitividad (CNIC), que dio origen a la Estrategia Nacional de Innovación para la Competitividad, Chile cuenta con una herramienta orientadora de políticas públicas y que releva el desarrollo del capital humano, la ciencia y la innovación.

Mediante este trabajo, de amplia participación y validación nacional, el Consejo definió cinco sectores productivos (clusters) de alto potencial de crecimiento para los próximos quince años, estableciendo brechas y oportunidades que Chile debe abordar, de manera de generar las condiciones necesarias para un mayor desarrollo productivo.

Así, por ejemplo, en materia de capital humano -técnico y avanzado-, la definición de los clusters por parte del CNIC implica también una priorización estratégica respecto de los conocimientos, destrezas y competencias que son necesarias de reforzar y del papel que, al respecto, les corresponde jugar a las instituciones de educación superior.

Con todo, hoy, luego de cinco años de iniciada la discusión, no existe información pública disponible respecto del grado de adecuación de la oferta y demanda de formación técnica de nivel superior en el país, ni de cómo ésta se comporta en relación con los clusters identificados. Tampoco si la oferta de formación de técnicos de nivel superior se ha visto impactada por esta priorización de sectores productivos o si se ha avanzado en la incorporación de técnicos de este tipo a la fuerza laboral del país. Tal ausencia de información impide la existencia de retroalimentación para orientar la política pública en educación y la necesaria articulación de este sector con el mundo laboral.

El estudio que a continuación se desarrolla busca responder a esas interrogantes. Para ello, en una primera parte, se contextualiza el tema a través de la revisión de la literatura existente, para luego ilustrar las consideraciones metodológicas adoptadas por los autores 
para abordar el tema. Posteriormente, se expone el análisis de los resultados y, para terminar, se presentan algunas reflexiones que, lejos de pretender poner un punto final en la materia, buscan aportar en la discusión sobre la articulación de la formación técnica de nivel superior con el mercado del trabajo.

\section{Marco de referencia}

\section{Formación técnica de nivel superior}

En Chile, la formación técnica de nivel superior se encuentra regulada a través de la Ley General de Educación (LGE) N²0.370, que la reconoce como un nivel de la educación superior a través del cual es posible acceder al título de técnico de nivel superior en las distintas áreas del conocimiento en que se dicta este tipo de programas. En el año 2009, un total de 185.269 estudiantes (CNED, 2010) cursaba formación técnica de nivel superior, lo que representa el 22\% de la matrícula total en educación superior.

La mayor oferta de programas de formación de nivel técnico se encuentra concentrada en los centros de formación técnica e institutos profesionales, sin perjuicio de que las universidades también pueden impartir carreras de este nivel. Así, los centros de formación técnica acumulan un $12 \%$ de la matrícula total, mientras que los institutos profesionales un $8 \%$ y las universidades un 2\% (CNED, 2010).

En términos generales, las carreras técnicas de nivel superior suelen caracterizarse por su corta duración -la que varía entre cuatro y seis semestres-, el menor costo de sus aranceles respecto de las carreras profesionales y la posibilidad que otorgan a sus titulados de lograr una rápida inserción laboral. En este contexto, se ha instalado en la discusión pública el interés por fomentar la ampliación de la matrícula en este nivel y el mejoramiento de la calidad de la oferta de formación, toda vez que se constata la existencia de un fenómeno de excesiva profesionalización de la educación postsecundaria, lo que ha incidido en una mayor proporción de profesionales que de técnicos, con los efectos que ello significa, tanto en impacto social como en el desarrollo productivo nacional (Bitrán, 2009). 
En el ámbito de las iniciativas impulsadas desde la política pública, se ha implementado un fondo de mejoramiento a través del programa MECESUP ${ }^{2}$ y un programa de becas para estudiantes desfavorecidos económicamente, que permite financiar parte o la totalidad del arancel anual. Además, se ha dado inicio a un programa de perfeccionamiento en el extranjero especialmente focalizado en técnicos, cuya selección se realiza sobre la base del mérito académico del postulante.

Según la comisión externa convocada por el Ministerio para realizar recomendaciones sobre política pública para el sector (MINEDUC, 2009), estas medidas han impactado favorablemente: "desde 2005 la tendencia al crecimiento de la matrícula técnica terciaria es más robusta, a partir de la consolidación de los sistemas de ayuda estudiantil". Así, en el periodo 2005-2009 (CNED, 2010), la matrícula total en carreras técnicas de nivel superior ha acumulado un crecimiento de un 79\%. En tanto, para el mismo periodo, las carreras no técnicas, impartidas por institutos profesionales y universidades, han incrementado su matrícula en un 30\%.

Con todo, si se considera el total de alumnos que estudian carreras de formación técnica de nivel superior, independientemente del tipo de institución en la que se encuentran matriculados, la participación de este nivel de formación representa un 22\% del total de los estudiantes en la educación terciaria, cifra que resulta aun menor si se considera que, además, este nivel exhibe una deserción importante, que alcanza al 40,7\% al término del primer año de estudios (MINEDUC, 2010).

Además del tema de la cobertura, nuevos desafíos se plantean para la formación técnica de nivel superior. En efecto, conceptos como empleabilidad de los egresados, pertinencia de la oferta y calidad de los programas de formación, entre otros, revisten especial importancia al momento de analizar este nivel de formación y su adecuación a las demandas del país, desde el horizonte de desarrollo que éste ha definido para los próximos años.

2 Mejoramiento de la Calidad de la Educación Superior. 
Desde este punto de vista, la comisión para la formación técnica concluye en su informe que la formación técnica de nivel superior "debe apoyar el aumento de la competitividad del país aportando capital humano en la cantidad y calidad requeridas por las empresas y sectores productivos. Esto implica que las demandas actuales y emergentes provenientes del mercado laboral y del desarrollo productivo del país constituyen un referente relevante para esta modalidad formativa, (sin perjuicio de también constatar que) la oferta de formación técnica de nivel superior presenta problemas de calidad y pertinencia respecto de los requerimientos del mundo del trabajo, y los esfuerzos efectuados hasta la fecha [...] no han logrado el impacto suficiente para revertir los problemas existentes" (MINEDUC, 2009).

\section{Estrategia Nacional de Innovación para la Competitividad}

El origen de la Estrategia Nacional de Innovación se remonta al año 2005, y encuentra sus cimientos en estudios y evidencias internacionales que subrayan la necesidad de que el modelo de desarrollo económico chileno deje de ser neutral e incorpore elementos de selectividad. A raíz de ello, durante 2005 y a través del primer Consejo Nacional de Innovación para la Competitividad (CNIC), se inicia un proceso de estudio e identificación de las áreas y sectores prioritarios de desarrollar en el país. Dicha tarea concluyó a principios del 2006 con la confección del documento "Lineamientos para una estrategia nacional de innovación para la competitividad".

En mayo de 2006 se constituye el actual CNIC como una entidad permanente de apoyo al desarrollo de la innovación en el país. Las principales tareas encomendadas al Consejo son continuar con las labores emprendidas por su antecesor y, principalmente, desarrollar una estrategia nacional de innovación para la competitividad. Para ello, el Consejo elaboró una metodología y desarrolló un proceso de trabajo que comprendió, entre sus principales actividades, la consulta a los actores del Sistema Nacional de Innovación acerca de sus análisis y perspectivas del estado actual y futuro de la innovación en Chile. 
Con la finalidad de contar con una visión de futuro que permitiera anticipar el escenario mundial y local para los próximos 15 años, identificando las capacidades específicas que los distintos sectores productivos -así como también el Estado- debían desarrollar, se realizó un estudio de competitividad en clusters nacionales. Como resultado de este proceso, se constituyó un mapa de los 11 sectores productivos más prometedores para la próxima década, entre los cuales se encuentran algunos en los que el país exhibe ventajas y otros que son emergentes.

De los 11 sectores identificados en un comienzo, el CNIC seleccionó ocho como prioritarios de abordar en el corto y mediano plazo, acorde con su mayor potencial competitivo para el país y la demanda internacional. A su vez, estos ocho sectores fueron agrupados en cinco clusters, los cuales, en definitiva, estructuran y orientan la política nacional de innovación y competitividad. Para cada uno de ellos, el CNIC hace entrega de una detallada hoja de ruta que orienta su desarrollo, incluyendo aspectos regulatorios, institucionales y actividades propiamente innovativas, así como sugerencias para el desarrollo de plataformas transversales como la investigación científica, la generación de capital humano específico y la disponibilidad de infraestructura física y digital, dejando en manos del ejecutivo la elaboración de la Política Nacional de Innovación y los planes de acción que materialicen la estrategia.

El estudio de los cinco clusters identificados se inició con el mapeo de la cadena de valor y un análisis de las tendencias globales de la industria, para luego continuar con el diagnóstico y análisis en profundidad de la situación del sector en Chile. Los clusters identificados son los siguientes (CONICYT, 2010):

1. Cluster alimentario: abarca los sectores frutícola, vitivinícola, de aves y cerdos, de carne bovina y ovina, y de alimentos procesados, con la finalidad de convertir a Chile en potencia alimentaria.

2. Cluster acuícola: incluye las industrias de crustáceos, peces, moluscos y plantas acuáticas, con el fin de promover el desarrollo económico y el incremento productivo del sector.

3. Cluster turismo de intereses especiales: el desafío es promover 
turísticamente a Chile en el extranjero, mejorar la oferta de actividades y productos, aumentar las capacidades de gestión y servicios de capital humano, e incrementar la asociatividad del sector.

4. Cluster servicios globales: busca incorporar nuevas tecnologías de la información, procesos de negocios, de conocimiento e innovación.

5. Cluster minero: pretende desarrollar una industria de servicios en torno a la minería, que posea conocimientos intensivos, conformando un sector que permita avanzar hacia una mayor sustentabilidad ambiental y de negocios.

De esta manera, la Estrategia Nacional de Innovación para la Competitividad se inscribe dentro del desafío de duplicar el Producto Interno Bruto per cápita al año 2020 -relevando temas de capital humano, ciencia e innovación- e identifica los principales obstáculos que pueden afectar su desarrollo. Asimismo, realiza recomendaciones específicas respecto de las acciones que debiera emprender el sector público con el fin de potenciar la innovación en el país, y sienta las bases para una renovada alianza público-privada.

Del análisis que el CNIC realiza de los distintos clusters, surge la constatación de requerimientos que son comunes y "pasan a constituir plataformas transversales, es decir, componentes de base cuyo desarrollo será determinante para enfrentar los desafíos de todos los sectores estudiados, y muy posiblemente, de la economía en su conjunto" (CNIC, 2008). Una de estas plataformas es el capital humano, ya que la calidad y disponibilidad de éste constituye una brecha crítica para capturar valor en los distintos clusters. En este sentido, la propuesta del Consejo se orienta a estimular una oferta de formación y capacitación más ajustada a las necesidades de la industria, que promueva la formación permanente y flexible.

Dentro de la necesidad de desarrollo del capital humano se puede identificar, por una parte, al capital humano avanzado, necesario para generar valor a través de la innovación en los mercados, pero, al mismo tiempo, se debe avanzar también en el desarrollo de capital humano en todos los niveles, especialmente en el nivel técnico, 
fortaleciendo los institutos profesionales y los centros de formación técnica, de manera de revertir la suerte de "pirámide invertida" que representa el sistema de educación superior en Chile en la actualidad (Bitrán, 2009).

\section{Consideraciones metodológicas}

El presente estudio considera la formación técnica de nivel superior en su totalidad, vale decir, son estudiados todos aquellos programas de formación conducentes a un título técnico de nivel superior, independientemente del tipo de institución que los ofrezca: centro de formación técnica, instituto profesional o universidad.

Los datos fueron extraídos del sistema INDICES del Consejo Nacional de Educación ${ }^{3}$. Se utilizan los datos del año 2004 (INDICES 2005) y del año 2009 (INDICES 2010), de manera de contar con información previa a la creación de la Estrategia Nacional de Innovación para la Competitividad (2005) y comparar el eventual impacto que ha tenido dicha estrategia en la oferta y la demanda de la formación técnica en Chile, transcurridos cinco años de su creación.

Para efectos del presente estudio, por oferta en la formación técnica se entiende el conjunto de programas ofrecidos por las distintas instituciones de educación superior. El término demanda, a su vez, hace referencia al interés de los estudiantes por dichos programas, cuya manifestación más clara, en este caso, es la matrícula.

La decisión de estudiar la demanda a través de la matrícula y no mediante el análisis de ocupación de las vacantes responde a dos criterios. En primer lugar, los datos de matrícula entregan una mayor confiabilidad que los de vacantes, los cuales, por lo general, son ingresados al sistema por las instituciones de modo referencial. Ello, es aun más relevante si se considera que la mayoría de las carreras de formación técnica, independientemente del tipo de institución a la que pertenezcan, realizan procesos de admisión no selectivos, que limitan la posibilidad de planificar las matrículas a través de la

3 INDICES es el sistema de información de educación superior que desarrolla y administra el Consejo Nacional de Educación (CNED), de acceso público, disponible en: www.cned.cl. 
determinación anticipada de vacantes. En segundo lugar, los datos de vacantes son establecidos por las propias instituciones de educación superior, sin que medie un patrón común respecto de la manera en que las determinan, siendo, por tanto, habitualmente, un reflejo de sus expectativas y no de la demanda real por dichos programas.

La utilización de las matrículas como expresión de la demanda, en cambio, es un dato validado y confiable dentro del sistema, y que representa el comportamiento de un actor fundamental dentro de éste: los estudiantes.

La clasificación de los distintos programas en los cinco clusters definidos por el CNIC se hizo a través de un proceso de filtrado multivariable, considerando para ello más de un atributo de la muestra, lo que permitió identificar 531 carreras distintas dentro de los 3.250 programas ${ }^{4}$ ofrecidos en todo el sistema para el año $2009^{5}$. De estas 531 carreras, 246 se clasificaron dentro de algún cluster. El mismo procedimiento se aplicó para el año 2004, identificándose 405 carreras distintas dentro de una oferta total de 1.751 programas de formación técnica. En este caso, 211 de las 405 carreras fueron clasificadas en alguno de los clusters.

Ahora bien, los datos que interesan para trabajar sobre la oferta y la demanda en formación técnica son los programas y no las carreras, ya que la apertura de un nuevo programa -aun cuando corresponda a una carrera ya existente-implica un crecimiento del sistema. En este marco la clasificación de las carreras por cluster se hizo extensiva a los programas. De esta manera, de los 3.250 programas de formación técnica que presentaba el sistema para el año 2009, 1.520 formaban parte de alguno de los cluster. Aplicado el mismo procedimiento para el año 2004, de los 1.751 programas de formación técnica en el sistema, 880 adscribían a alguno.

4 La diferencia entre carreras y programas se explica porque una misma carrera puede ser ofrecida en distintos programas, según la institución que lo imparta y la sede o jornada en que se dicte.

5 Se excluyeron de este estudio los programas ofrecidos por instituciones de la Fuerzas Armadas, ya que responden a una lógica distinta al desarrollo productivo del país. 
Tabla n..$^{\circ} 1$

\begin{tabular}{|l|c|c|c|c|c|c|}
\hline & $\begin{array}{c}\text { Cluster servicios } \\
\text { globales }\end{array}$ & $\begin{array}{c}\text { Cluster } \\
\text { alimentario }\end{array}$ & $\begin{array}{c}\text { Cluster } \\
\text { acuícola }\end{array}$ & $\begin{array}{c}\text { Cluster } \\
\text { turismo }\end{array}$ & $\begin{array}{c}\text { Cluster } \\
\text { minero }\end{array}$ & Total \\
\hline Año 2004 & 579 & 82 & 9 & 185 & 25 & 880 \\
\hline Ano 2009 & 1075 & 127 & 28 & 245 & 45 & 1520 \\
\hline
\end{tabular}

Fuente: elaboración propia a partir de los datos del sistema INDICES del Consejo Nacional de Educación.

Si bien se constata la existencia de algunos estudios en materias relacionadas, tal como los realizados por el Instituto de Desarrollo Local y Regional (IDER) de la Universidad de La Frontera (UFRO, 2008) (AUR, 2009), éstos no poseen foco específico en la formación técnica ni analizan la oferta y demanda en los términos aquí utilizados. En consecuencia, no es posible encontrar algún tipo de clasificación exhaustiva de los distintos programas de formación técnica impartidos en el país, en relación con los clusters definidos por el CNIC. Por lo tanto, el presente estudio propone un modelo operativo de clasificación, basándose en las orientaciones antes mencionadas. Para ello, se articulan los aportes realizados por el Sistema Bicentenario Becas Chile -donde se definen las áreas de formación privilegiadas para Pasantías de Perfeccionamiento de Competencias Técnicas en el Extranjero para cada uno de los cinco clusters- junto con las definiciones productivas relativas a cada área de priorización entregadas por CORFO y el Consejo Nacional de Innovación para la Competitividad.

Sobre la base de las orientaciones mencionadas y de acuerdo con los objetivos del estudio, se clasificaron sólo aquellos programas que presentaban una relación directa con el cluster, dejando fuera de la clasificación aquellos casos que no cumplían con este criterio de exhaustividad ${ }^{6}$. Asimismo, la pertinencia de los programas en relación con los clusters se consideró de manera excluyente, vale decir, las carreras se asocian sólo a un cluster, quedando con ello impedidas de adscribir a otro. Por lo tanto, para aquellos interesados en profundizar o explorar otras áreas en el estudio de la relación entre la oferta y demanda de los programas de formación técnica en Chile y la Estrategia Nacional de Innovación, puede serles útil una revisión más extensiva que la clasificación propuesta.

6 Véase anexo n. ${ }^{\circ}$ 1: Carreras por cluster. Año 2004 y 2009. 
En consideración a la existencia de diversos lineamientos que orientan el desarrollo productivo regional, tales como el Fondo de Innovación para la Competitividad, los programas de Mejoramiento para la Competitividad, los Nodos Tecnológicos y el Programa Territorial Integrado (UFRO, 2008), entre otros, para la definición de las estrategias productivas regionales, se optó por emplear la clasificación que CORFO expone al respecto. Ello, debido a que esa institución "recibió de parte del Comité de Ministros para la Innovación el mandato para desarrollar el Programa Nacional de Clusters, brindando soporte metodológico, financiero y operativo a las secretarías ejecutivas de los consejos estratégicos público-privados de los clusters" (CORFO, 2010). Además, dada su cobertura territorial de carácter nacional y presencia regional, dota de un tratamiento conceptual similar en cada región. Junto con ello, considerando el cambio político-geográfico acaecido en el país el año 2007 y de manera de permitir la comparación, para el 2009 se reclasificaron las regiones, manteniendo el sistema de regionalización vigente al año 2004. De esta manera, para el análisis del año 2009, las regiones de Arica y Tarapacá se agrupan como si conformaran la Región "Arica-Tarapacá". El mismo procedimiento se utilizó con las regiones De Los Ríos y Los Lagos, agrupándose en la Región de "Los Ríos-Los Lagos".

\section{Análisis de resultados}

\section{Caracterización de la formación técnica de nivel superior en Chile (2004-2009)}

El sistema de educación superior ha experimentado un importante crecimiento en los últimos años y la formación técnica no ha sido la excepción, mostrando altos niveles de crecimiento, tanto en la oferta como en la demanda, e indistintamente del tipo de institución que la imparta (centros de formación técnica, institutos profesionales o universidades).

Como se observa en la tabla n. ${ }^{\circ} 1$, para el año 2004, la oferta de programas de formación técnica de nivel superior era de 1.751, lo que representaba el $35 \%$ del total de la oferta del sistema de educación superior. Para el año 2009, en tanto, esta cifra asciende a 
3.250 programas, equivalentes al $45 \%$ del total del sistema. Estas cifras evidencian una masificación importante de la oferta de formación en este nivel, alcanzando un crecimiento del 53\% para el periodo 200420097, enmarcándose dentro de la tendencia de masificación de la educación superior en Chile (Brunner, 2006).

Tabla n. ${ }^{\circ} 2$

\begin{tabular}{|c|c|c|c|c|}
\hline Año & $\begin{array}{c}\text { N. }{ }^{\circ} \text { de } \\
\text { programas }\end{array}$ & $\begin{array}{c}\text { Participación respecto del } \\
\text { total de programas }\end{array}$ & $\begin{array}{c}\text { Matrícula } \\
\text { total }\end{array}$ & $\begin{array}{c}\text { Participación respecto de la } \\
\text { matrícula total }\end{array}$ \\
\hline 2004 & 1.751 & $35 \%$ & 83.902 & $16 \%$ \\
\hline 2009 & 3.250 & $45 \%$ & 178.033 & $24 \%$ \\
\hline
\end{tabular}

Fuente: Elaboración propia a partir de los datos del sistema INDICES del Consejo Nacional de Educación.

En cuanto a la demanda por este tipo de programas, representada en la matrícula total, se observa en la tabla n. ${ }^{\circ} 2$ que el crecimiento también ha sido importante, aumentando a más del doble en términos de estudiantes matriculados, y pasando de representar un 16\% de la matrícula total del sistema el año 2004 a un 24\% el 2009.

\section{Caracterización de la oferta de formación técnica de nivel superior según cluster}

Los datos presentados en la tabla n. ${ }^{\circ} 2$ indican la distribución actual (2009) de la oferta de programas de formación técnica de nivel superior según el cluster al cual adscriben.

7 Si se analiza el crecimiento de las carreras de formación técnica, independientemente del número de programas al que dan origen, en el mismo periodo, se observa que ellas sólo crecieron un $24 \%$, lo que genera dudas respecto del dinamismo en el surgimiento de nuevas carreras vinculadas a sectores económicos emergentes, más aun si se considera que es probable que esta cifra sea incluso menor, dada la imposibilidad del sistema para diferenciar entre carreras similares pero con ligeros cambios en su denominación (por ejemplo: Técnico en Contabilidad y Técnico en Gestión Contable). 
160 PERTINENCIA DE LA FORMACIÓN TÉCNICA DE NIVEL SUPERIOR EN RELACIÓN CON LA ESTRATEGIA NACIONAL DE INNOVACIÓN PARA LA COMPETITIVIDAD - Pablo Carrasco, Juan Pablo Venables

Tabla n. ${ }^{\circ} 3$

\begin{tabular}{|l|c|c|}
\hline Cluster & $\begin{array}{c}\mathrm{N}^{\circ}{ }^{\circ} \text { de } \\
\text { programas }\end{array}$ & $\begin{array}{c}\text { Participación respecto de los programas clasificados al } \\
\text { interior de algún cluster }\end{array}$ \\
\hline Acuicultura & 28 & $1,7 \%$ \\
\hline Alimentario & 127 & $8,3 \%$ \\
\hline Minería & 45 & $3,0 \%$ \\
\hline Servicios globales & 1.075 & $71 \%$ \\
\hline Turismo & 245 & $16 \%$ \\
\hline Total general & 1.520 & $100 \%$ \\
\hline
\end{tabular}

Fuente: elaboración propia a partir de los datos del sistema INDICES del Consejo Nacional de Educación.

En la tabla n. ${ }^{\circ} 3$ se observa una clara preeminencia del cluster de servicios globales por sobre el resto, representando más de dos tercios de la oferta total de programas de formación técnica clasificados dentro de algún cluster. Asimismo, los programas dedicados a industrias de alta especialización y de importante dinamismo económico en el país, como la acuicultura y la minería, presentan una baja oferta de formación técnica de nivel superior, la que alcanza, respectivamente, un $1,7 \%$ y un $3 \%$ del total de este tipo de programas.

2.1. Distribución de la oferta de programas según cluster y tipo de institución de educación superior (IES)

Al analizar la distribución de la oferta de programas de formación técnica que tributan a alguno de los clusters, para el año 2009, se puede observar que los distintos tipos de IES cuentan con una oferta que considera carreras en todos los clusters, concentrándose la mayor parte de ella -como era de esperarse según lo señalado con la tabla anterior- en el cluster de servicios globales.

Tabla n. ${ }^{\circ} 4$

\begin{tabular}{|l|c|c|c|c|}
\hline Cluster & Universidades & $\begin{array}{c}\text { Institutos } \\
\text { Profesionales }\end{array}$ & $\begin{array}{c}\text { Centros de } \\
\text { Formación Técnica }\end{array}$ & Total \% \\
\hline Acuícola & $14(50 \%)$ & $10(36 \%)$ & $4(14 \%)$ & $100 \%$ \\
\hline Alimentario & $19(15 \%)$ & $34(27 \%)$ & $74(58 \%)$ & $100 \%$ \\
\hline Minería & $16(36 \%)$ & $7(16 \%)$ & $22(49 \%)$ & $100 \%$ \\
\hline Servicios globales & $129(12 \%)$ & $356(33 \%)$ & $590(55 \%)$ & $100 \%$ \\
\hline Turismo & $21(9 \%)$ & $83(34 \%)$ & $141(58 \%)$ & $100 \%$ \\
\hline TOTAL & $199(13 \%)$ & $490(32 \%)$ & $831(55 \%)$ & $100 \%$ \\
\hline
\end{tabular}

Fuente: elaboración propia a partir de los datos del sistema INDICES del Consejo Nacional de Educación. 
Como se observa en la tabla n. ${ }^{\circ} 4$, sólo un $13 \%$ de los programas de formación técnica que adscriben a alguno de los clusters es impartido por universidades, en tanto que los centros de formación técnica concentran el 55\% de la oferta de este tipo de formación. Este dato no deja de ser interesante, pues si bien se relaciona con cierta especialización institucional para impartir programas de formación técnica de nivel superior y con las atribuciones que la Ley General de Educación (LGE) confiere a cada tipo de institución de educación superior, a su vez, releva la importancia que tendrían los centros de formación técnica en una eventual política pública relativa al desarrollo de este tipo de formación.

En este contexto, se pueden destacar varias situaciones. En primer lugar, el hecho de que el cluster acuícola es el único grupo cuyos programas se concentran en universidades y no en centros de formación técnica. Asimismo, mientras las universidades ofrecen el $36 \%$ de los programas de minería, los centros de formación técnica concentran el $58 \%$ de la oferta de programas vinculados a los clusters alimentario y turismo. Finalmente, en ninguno de los cinco grupos los institutos profesionales logran un desempeño mayor que los otros tipos de instituciones.

2.2. Distribución de la oferta de programas según régimen de IES y cluster

Con el objeto de identificar cómo se distribuye la oferta de formación técnica en los programas incluidos en los clusters, se clasificó operacionalmente a las instituciones de educación superior en tres grupos: autónoma acreditada, autónoma no acreditada y no autónoma. Ello, con el objeto de medir si la autonomía institucional y los procesos de acreditación poseen algún impacto en la oferta de carreras y la relación de éstas con los clusters productivos. 
162 PERTINENCIA DE LA FORMACIÓN TÉCNICA DE NIVEL SUPERIOR EN RELACIÓN CON LA ESTRATEGIA NACIONAL DE INNOVACIÓN PARA LA COMPETITIVIDAD - Pablo Carrasco, Juan Pablo Venables

Tabla n. ${ }^{\circ} 5$

\begin{tabular}{|l|c|c|c|c|c|c|}
\hline Cluster & $\begin{array}{c}\text { Autónoma } \\
\text { acreditada }\end{array}$ & $\%$ & $\begin{array}{c}\text { Autónoma } \\
\text { no acreditada }\end{array}$ & $\%$ & $\begin{array}{c}\text { No } \\
\text { autónoma }\end{array}$ & $\%$ \\
\hline Acuícola & 16 & $57 \%$ & 11 & $39 \%$ & 1 & $4 \%$ \\
\hline Alimentario & 94 & $74 \%$ & 19 & $15 \%$ & 14 & $11 \%$ \\
\hline Minería & 35 & $78 \%$ & 7 & $16 \%$ & 3 & $7 \%$ \\
\hline Servicios globales & 697 & $65 \%$ & 240 & $22 \%$ & 138 & $13 \%$ \\
\hline Turismo & 169 & $69 \%$ & 59 & $24 \%$ & 17 & $7 \%$ \\
\hline TOTAL & 1011 & $67 \%$ & 336 & $22 \%$ & 173 & $11 \%$ \\
\hline
\end{tabular}

Fuente: elaboración propia a partir de los datos del sistema INDICES del Consejo Nacional de Educación.

Como se desprende de la tabla, el mayor número de programas clasificados en algún cluster es ofrecido por instituciones autónomas, oferta que, en su conjunto, representa al 89\% del total. Esta situación puede explicarse dado que la mayoría de las IES existentes en el país posee tal condición y, precisamente producto de su autonomía, para ellas la apertura de nuevos programas de formación sólo está condicionada a la decisión institucional, sin que existan limitantes regulatorias de organismos externos.

En cuanto a la oferta de formación de las IES autónomas, se puede observar que un $67 \%$ del total de los programas de formación técnica pertenecientes a los clusters es dictado por instituciones acreditadas, mientras que el $22 \%$ corresponde a instituciones no acreditadas (ya sea porque no se han presentado a este proceso voluntario o se les ha negado tal certificación). Cabe mencionar que la importancia del dato radica en que la acreditación institucional representa una suerte de sello de calidad de la gestión de una IES, por tanto, es el único indicador actualmente disponible respecto del "conjunto de políticas y mecanismos institucionales destinados a asegurar la calidad de la formación conducente a título, principalmente en cuanto a las decisiones acerca de la oferta de carreras y su pertinencia, apertura de sedes, definición y revisión de perfiles de egreso, diseño curricular, asignación de recursos, seguimiento de los resultados", entre otros (CNA, 2010). Ello adquiere mayor relevancia si se considera que, en el nivel de acreditación de carreras, esta misma certificación de calidad, a julio de 2010, sólo la poseen 29 programas técnicos de nivel superior (CNA, 2010), lo que representa un $0,9 \%$ del total de programas de formación técnica de nivel superior del país. 
Por último, las instituciones de educación superior que aún no han obtenido la autonomía institucional imparten un $11 \%$ de la oferta total de programas pertenecientes a un cluster.

3. Caracterización de la demanda por formación técnica de nivel superior según cluster

En términos generales, la demanda por programas de formación técnica de nivel superior presenta una alta sintonía con la oferta antes señalada. En ambos casos, oferta y demanda presentan la misma relación ordinal entre los clusters.

Tabla n. ${ }^{\circ} 6$

\begin{tabular}{|l|c|c|}
\hline Cluster & $N^{\circ}$ de matrículas & $\begin{array}{c}\text { Participación respecto de los programas clasificado al } \\
\text { interior de algún cluster }\end{array}$ \\
\hline Acuicultura & 553 & $0,8 \%$ \\
\hline Minería & 2116 & $3,1 \%$ \\
\hline Alimentario & 4923 & $7,3 \%$ \\
\hline Turismo & 14.480 & $21,4 \%$ \\
\hline Servicios globales & 45.594 & $67,4 \%$ \\
\hline Total general & 67.666 & $100 \%$ \\
\hline
\end{tabular}

Fuente: elaboración propia a partir de los datos del sistema INDICES del Consejo Nacional de Educación.

La concordancia evidenciada entre oferta y demanda de programas clasificados dentro de algún cluster muestra que las industrias de alta especialización, como la acuicultura y la minería, mantienen un bajo nivel de desarrollo en términos de formación técnica de nivel superior.

4. Comportamiento de la oferta y la demanda para el periodo 2004-2009, en programas de formación técnica clasificados dentro de los clusters

\subsection{Respecto de la oferta}

Con el fin de identificar el comportamiento de la oferta de formación de programas técnicos de nivel superior, se revisó la distribución de programas según cluster para el año 2004 y se comparó con similar información del año 2009, como se observa en la tabla n. ${ }^{\circ}$ 6. De allí se puede desprender que el comportamiento de los programas de 
formación técnica que tributan a alguno de los clusters experimentaron un importante crecimiento, independientemente del cluster.

Durante el mismo periodo, la oferta de formación técnica de nivel superior se incrementó en un $86 \%$, crecimiento que, para efecto de este estudio, es considerado vegetativo. Con este concepto se hace referencia al crecimiento de todos los programas de formación técnica impartidos en el país, independientemente de los cluster. Por lo tanto, cumple la función de variable de control, permitiendo despejar el crecimiento efectivo por cluster de aquel que responde a variables anexas.

Tabla n. ${ }^{\circ} 7$

\begin{tabular}{|l|c|c|c|c|}
\hline Cluster & $\begin{array}{c}\text { Programas } \\
2004\end{array}$ & $\begin{array}{c}\text { Programas } \\
2010\end{array}$ & $\begin{array}{c}\text { Crecimiento } \\
2004-2009\end{array}$ & $\begin{array}{c}\text { Crecimiento efectivo } \\
\text { (descontado el } \\
\text { crecimiento vegetativo) }\end{array}$ \\
\hline Acuícola & 9 & 28 & $211 \%$ & $126 \%$ \\
\hline Alimentario & 82 & 127 & $55 \%$ & $-31 \%$ \\
\hline Minería & 25 & 45 & $80 \%$ & $-6 \%$ \\
\hline Servicios globales & 579 & 1075 & $86 \%$ & $0 \%$ \\
\hline Turismo & 185 & 245 & $32 \%$ & $-53 \%$ \\
\hline Total & 880 & 1.520 & $73 \%$ & $-13 \%$ \\
\hline Total sistema programas de FTP & 1.751 & 3.250 & $86 \%$ & \\
\hline
\end{tabular}

Fuente: elaboración propia a partir de los datos del sistema INDICES del Consejo Nacional de Educación.

Como se desprende de la tabla, el cluster acuícola presenta el crecimiento más significativo del periodo, incrementando la oferta de programas de formación técnica en un $211 \%$, seguido por servicios globales y minería. Sin embargo, dado el bajo número de programas ofertados el año 2004 en los clusters acuícola y minería, el crecimiento alcanzado por ambos adquiere cierta relatividad y no es necesariamente representativo de un mayor énfasis por dotarlos de un especial dinamismo.

A su vez, al comparar el crecimiento de cada cluster con el crecimiento vegetativo, se observa que el desarrollo de los programas de formación técnica que se encuentran dentro de alguno de los clusters también adquiere cierta relatividad. En efecto, aplicando dicha variable de control, se observa que sólo el cluster acuícola 
se incrementó por sobre el crecimiento del total de programas de formación técnica dentro del sistema; el resto de los clusters presenta un crecimiento negativo o neutro.

En este contexto, se observa también que, en total, la oferta de programas de formación técnica que se encuentra dentro de alguno de los clusters creció un 13\% menos que el total de programas de formación técnica del sistema.

\subsection{Respecto de la demanda}

Comparando la matrícula de los programas que se encuentran dentro de alguno de los cluster llama la atención que, en términos generales, no se observa concordancia con la oferta.

Tabla n. ${ }^{\circ} 8$

\begin{tabular}{|l|c|c|c|c|}
\hline Cluster & $\begin{array}{c}\text { Matrícula } \\
2004\end{array}$ & $\begin{array}{c}\text { Matrícula } \\
2009\end{array}$ & $\begin{array}{c}\text { Crecimiento } \\
2004-2009\end{array}$ & $\begin{array}{c}\text { Crecimiento efectivo } \\
\text { (descontado el crecimiento } \\
\text { vegetativo) }\end{array}$ \\
\hline Acuícola & 525 & 553 & $5 \%$ & $-107 \%$ \\
\hline Alimentario & 4.259 & 4.923 & $16 \%$ & $-97 \%$ \\
\hline Minería & 700 & 2.116 & $202 \%$ & $90 \%$ \\
\hline Servicios globales & 23.289 & 45.594 & $96 \%$ & $-16 \%$ \\
\hline Turismo & 11.098 & 14.480 & $30 \%$ & $-82 \%$ \\
\hline Total & 39.871 & 67.666 & $70 \%$ & $-42 \%$ \\
\hline Total sistema programas de FTP & 83.902 & 178.033 & $112 \%$ & \\
\hline
\end{tabular}

Fuente: elaboración propia a partir de los datos del sistema INDICES del Consejo Nacional de Educación.

Como se desprende de la tabla, todos los clusters experimentan un crecimiento de su matrícula, destacando minería con un 202\%. Ahora bien, llama la atención que el cluster acuícola, que presentaba el mayor crecimiento en cuanto a oferta de programas, sea, a su vez, el con menor crecimiento en cuanto a las matrículas, sobre todo considerando que el crecimiento vegetativo del total de programas de formación técnica para el periodo es de 112\%.

Asimismo, comparando la demanda con la oferta y controlando los resultados con el crecimiento vegetativo, se observa que el cluster acuícola pasa de ser el único con un crecimiento de la oferta por sobre el crecimiento vegetativo, a ser el con menor crecimiento de 
demanda para el mismo periodo. Por el contrario, el cluster minería presenta un importante crecimiento de la matrícula por sobre el crecimiento vegetativo, erigiéndose como el único cluster en crecer en este sentido.

Por último, es destacable también el hecho de que, si bien la oferta del total de programas de formación técnica adscritos a algún cluster tuvo un decrecimiento de 13\% para el periodo 2004-2009, la matrícula para este tipo de programas creció aun menos (-42\%).

\subsection{Análisis por región}

Un aspecto distintivo dentro de la estrategia de innovación tiene relación con su especificación a nivel regional, puesto que, por un lado, determina el contexto de desarrollo de las distintas empresas, y por otro, afecta la disponibilidad de algunos bienes públicos esenciales, como la investigación y el capital humano.

Como sostiene el CNIC, esta consideración es particularmente importante al momento de definir e implementar programas para el desarrollo de los distintos clusters: "en efecto, las actividades vinculadas con estos clusters o sectores se localizan y tienen impacto, en su mayoría, en las regiones donde se encuentran sus materias primas" (CNIC, 2008). Asimismo, agrega que la responsabilidad en la provisión de bienes públicos que generen ambientes favorables para el desarrollo de los clusters debiera concentrarse, principalmente, en instituciones regionales, ya que son ellas las que cuentan con un conocimiento más cercano y certero respecto de la realidad de las distintas actividades productivas del territorio. En consecuencia, "esto hace necesario que la estrategia nacional de innovación incluya políticas regionales diferenciadas, asegurando la participación de las propias regiones" (CNIC, 2008).

En este contexto, cada región ha establecido en su agenda de desarrollo productivo distintas priorizaciones de aquellos sectores de la economía en los cuales deben focalizar sus esfuerzos (UFRO, 2009). A continuación, se analiza el desarrollo de la oferta y la demanda de formación técnica por región, según la priorización de los clusters definida por cada una de ellas. 


\subsubsection{Oferta de programas}

Respecto de la oferta de programas de formación técnica por región, presentada en la tabla n. ${ }^{\circ}$, en términos generales, se puede observar que, para el año 2004, de los 880 programas de formación técnica que se clasifican dentro de algún cluster, 341 (39\%) son ofertados en la Región Metropolitana. Si a ello se suman las regiones del Biobío y De Valparaíso (agrupando los tres grandes centros urbanos del país), el número crece a 545, equivalente al $62 \%$ de la oferta de programas que corresponde a alguno de los clusters.

Similar situación se observa para el año 2009; de los 1.520 programas de formación técnica clasificados dentro de algún cluster, 523 (34\%) se encuentran en la Región Metropolitana. Sumando las regiones de Valparaíso y Biobío, el número asciende a 909 programas, equivalentes al $60 \%$ de la oferta total de programas dentro de algún cluster en el país.

No obstante, como se desprende de la última columna de la tabla $n .{ }^{\circ}$, las distintas regiones presentan un porcentaje de oferta de programas perteneciente a algún cluster en relación con la oferta total de programas de formación técnica de la región, bastante similar. El promedio para el año 2004 es de 49\%, mientras que para el 2009 esta cifra baja a 47\%. Destacan los casos de las regiones de Tarapacá-Arica $\left(1^{a}\right)$ y Atacama (3a), que en el periodo 2004-2009 presentan una baja considerable de la oferta de programas pertenecientes a alguno de los clusters (16\% y 13\% respectivamente). En la situación contraria, destacan las regiones del Maule $\left(7^{\mathrm{a}}\right)$ y Aysén $\left(11^{\mathrm{a}}\right)$, con un aumento de los programas que tributan a alguno de los clusters en relación con el total, de un $11 \%$ y un $14 \%$, respectivamente. 
168 PERTINENCIA DE LA FORMACIÓN TÉCNICA DE NIVEL SUPERIOR EN RELACIÓN CON LA ESTRATEGIA NACIONAL DE INNOVACIÓN PARA LA COMPETITIVIDAD - Pablo Carrasco, Juan Pablo Venables

Tabla n. ${ }^{\circ} 9$

\begin{tabular}{|c|c|c|c|c|c|c|c|c|}
\hline \multirow[t]{2}{*}{ Región } & \multirow[t]{2}{*}{ Año } & \multicolumn{6}{|c|}{ Cluster } & \multirow{2}{*}{$\begin{array}{l}\% \text { del total } \\
\text { del sistema } \\
\text { representado } \\
\text { por cluster }\end{array}$} \\
\hline & & $\begin{array}{l}\text { Servicios } \\
\text { globales }\end{array}$ & Alimentario & Acuícola & Turismo & Minero & $\begin{array}{l}\text { Total } \\
\text { cluster }\end{array}$ & \\
\hline \multirow{2}{*}{$1^{\circ}$} & 2004 & 24 & 1 & 0 & 13 & 3 & 41 & $59 \%$ \\
\hline & 2009 & 34 & 2 & 0 & 7 & 6 & 49 & $43 \%$ \\
\hline \multirow{2}{*}{$2^{\circ}$} & 2004 & 25 & 0 & 0 & 10 & 4 & 39 & $45 \%$ \\
\hline & 2009 & 41 & 1 & 0 & 12 & 13 & 67 & $45 \%$ \\
\hline \multirow{2}{*}{$3^{\circ}$} & 2004 & 9 & 1 & 0 & 3 & 1 & 14 & $50 \%$ \\
\hline & 2009 & 16 & 1 & 1 & 2 & 2 & 22 & $37 \%$ \\
\hline \multirow{2}{*}{$4^{\circ}$} & 2004 & 27 & 4 & 0 & 9 & 0 & 40 & $46 \%$ \\
\hline & 2009 & 59 & 8 & 1 & 11 & 5 & 84 & $53 \%$ \\
\hline \multirow{2}{*}{$5^{\circ}$} & 2004 & 83 & 13 & 2 & 23 & 2 & 123 & $51 \%$ \\
\hline & 2009 & 149 & 13 & 2 & 31 & 4 & 199 & $44 \%$ \\
\hline \multirow{2}{*}{$6^{\circ}$} & 2004 & 31 & 7 & 0 & 4 & 1 & 43 & $54 \%$ \\
\hline & 2009 & 47 & 15 & 0 & 7 & 2 & 71 & $48 \%$ \\
\hline \multirow{2}{*}{$7^{\circ}$} & 2004 & 17 & 7 & 0 & 6 & 0 & 30 & $38 \%$ \\
\hline & 2009 & 58 & 20 & 0 & 5 & 2 & 85 & $49 \%$ \\
\hline \multirow{2}{*}{$8^{\circ}$} & 2004 & 45 & 10 & 0 & 24 & 2 & 81 & $40 \%$ \\
\hline & 2009 & 131 & 14 & 0 & 40 & 2 & 187 & $40 \%$ \\
\hline \multirow{2}{*}{$9^{\circ}$} & 2004 & 20 & 3 & 1 & 8 & 2 & 34 & $51 \%$ \\
\hline & 2009 & 42 & 9 & 1 & 8 & 2 & 62 & $45 \%$ \\
\hline \multirow{2}{*}{$10^{\circ}$} & 2004 & 35 & 8 & 3 & 19 & 1 & 66 & $49 \%$ \\
\hline & 2009 & 67 & 14 & 15 & 22 & 1 & 119 & $49 \%$ \\
\hline \multirow{2}{*}{$11^{\circ}$} & 2004 & 3 & 0 & 0 & 2 & 0 & 5 & $42 \%$ \\
\hline & 2009 & 5 & 1 & 1 & 2 & 0 & 9 & $56 \%$ \\
\hline \multirow{2}{*}{$12^{\circ}$} & 2004 & 11 & 0 & 1 & 10 & 1 & 23 & $52 \%$ \\
\hline & 2009 & 27 & 1 & 6 & 9 & 0 & 43 & $57 \%$ \\
\hline \multirow{2}{*}{$\mathrm{RM}$} & 2004 & 249 & 28 & 2 & 54 & 8 & 341 & $55 \%$ \\
\hline & 2009 & 399 & 28 & 1 & 89 & 6 & 523 & $50 \%$ \\
\hline
\end{tabular}

Fuente: elaboración propia a partir de los datos del sistema INDICES del Consejo Nacional de Educación.

Nota: el achurado representa los clusters que son priorizados por cada región.

Ahora bien, lo que resulta más interesante respecto de esta tabla tiene relación con la priorización que cada región hace de los clusters (CORFO, 2010), como se grafica en las zonas achuradas. De esta manera, se puede observar, por ejemplo, que mientras los clusters acuícola y minero se concentran geográficamente en los extremos del país, los clusters alimentario y turismo de intereses especiales se encuentran indistintamente en casi todas las regiones. Asimismo, el de servicios globales está presente exclusivamente en los grandes centros urbanos. 
En relación con lo descrito, llama la atención que en todas las regiones, y tanto para el año 2004 como para el 2009, el cluster de servicios globales concentra la mayor cantidad de oferta. Si se considera, además, que sólo tres de las 13 regiones priorizan este cluster, la situación llama aun más la atención. Junto con ello, en términos de promedio por región, este cluster es el que presenta un mayor crecimiento para el periodo 2004-2009.

Por otro lado, es notorio que, con excepción de las regiones de Los Lagos-Los Ríos $\left(10^{a}\right)$ y la de Magallanes $\left(12^{a}\right)$, que ostentan un importante crecimiento para el periodo, el cluster acuícola presenta una oferta muy baja o inexistente, incluso en aquellas regiones que señalan al sector acuícola como prioritario. Asimismo, las regiones

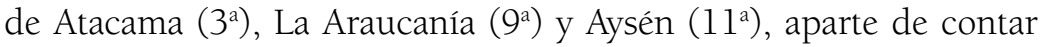
con una oferta de formación técnica muy baja en el sector, presentan un crecimiento mínimo o nulo para el periodo 2004-2009, pese a señalarlo como prioritario para la región.

Las regiones que priorizaron el cluster alimentario, por su parte, presentan situaciones disímiles. Mientras las regiones de Tarapacá-

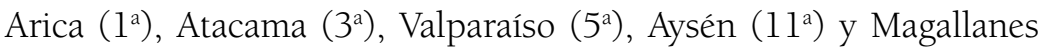
$\left(12^{a}\right)$ cuentan con un crecimiento muy bajo en términos absolutos ${ }^{8}$, o incluso nulo en algunos casos, las regiones de Coquimbo (4a), O’Higgins (6 $6^{a}$, Del Maule (7a), Del Biobío ( $8^{a}$ ), La Araucanía (9a) y Los Lagos-Los Ríos $\left(10^{a}\right)$, presentan un crecimiento importante para el periodo, duplicando y, en algunos casos, triplicando el número de programas de formación técnica vinculados al sector alimentario.

El cluster turismo de intereses especiales es priorizado por todas las regiones, con excepción de la Metropolitana (RM) y Atacama (3). Sin embargo, no presenta un nivel homogéneo de desarrollo de la oferta; mientras que en algunas de las regiones el número de programas no aumentó, o incluso disminuyó, en otras regiones se observa un aumento en este sentido, destacando las regiones de

8 Con crecimiento en términos reales se hace referencia a un crecimiento que tenga un impacto real en la oferta, pues, se da el caso de regiones donde para el periodo 2004-2009 se creció de un programa a dos, lo que, si bien en términos porcentuales representa un crecimiento de un $100 \%$, en término reales no resulta significativo. 
Valparaíso ( $5^{a}$ ) y Biobío ( $8^{a}$ ), cuyo crecimiento de oferta alcanza los 8 y 16 programas, respectivamente.

Por último, el sector minero es el cluster que menos regiones priorizaron; sólo cuatro de las 13. Se ubica en el extremo norte y en la región más austral del país y, con excepción de esta última, presenta un crecimiento importante para el periodo 2004-2009, sobre todo en la Región de Antofagasta ( $2^{a}$ ), donde -coherentemente con la zona geográfica en que se concentra la mayor cantidad de yacimientos mineros (SERNAGEOMIN, 2010)- la oferta de formación técnica triplicó el número de programas que tributan a este sector.

Tabla n. ${ }^{\circ} 10$

\begin{tabular}{|c|c|c|c|}
\hline Región & Crecimiento en cluster 2004-2009 & Crecimiento vegetativo 2004-2009 & Diferencial \\
\hline $1^{\mathrm{a}}$ & $20 \%$ & $65 \%$ & -45 \\
\hline $2^{\mathrm{a}}$ & $72 \%$ & $72 \%$ & 0 \\
\hline $3^{\mathrm{a}}$ & $57 \%$ & $111 \%$ & -54 \\
\hline $4^{\mathrm{a}}$ & $110 \%$ & $83 \%$ & 27 \\
\hline $5^{\mathrm{a}}$ & $62 \%$ & $89 \%$ & -27 \\
\hline $6^{\mathrm{a}}$ & $65 \%$ & $85 \%$ & -20 \\
\hline $7^{\mathrm{a}}$ & $183 \%$ & $122 \%$ & 62 \\
\hline $8^{\mathrm{a}}$ & $131 \%$ & $128 \%$ & 3 \\
\hline $9^{\mathrm{a}}$ & $82 \%$ & $106 \%$ & -24 \\
\hline $10^{\mathrm{a}}$ & $80 \%$ & $80 \%$ & 0 \\
\hline $11^{\mathrm{a}}$ & $80 \%$ & $33 \%$ & 47 \\
\hline $12^{\mathrm{a}}$ & $87 \%$ & $73 \%$ & 14 \\
\hline $\mathrm{RM}$ & $53 \%$ & $70 \%$ & -17 \\
\hline
\end{tabular}

Fuente: elaboración propia a partir de los datos del sistema INDICES del Consejo Nacional de Educación.

Ahora bien, el análisis hasta aquí expuesto presenta, en términos generales, un crecimiento de la oferta de programas de formación técnica que se encuentran dentro de algún cluster en todas las regiones. Sin embargo, al controlar dichos resultados a través de la variable de crecimiento vegetativo del total de programas de formación técnica, se observa que sólo cinco de las 13 regiones presenta un crecimiento de la oferta de programas pertenecientes a algún cluster superior al crecimiento vegetativo.

Asimismo, seis de las 13 regiones presentan un diferencial de crecimiento negativo, destacando las regiones de Tarapacá-Arica 
$\left(1^{a}\right)$ y Atacama ( $\left.3^{a}\right)$. Con todo, las cifras son elocuentes respecto de la sintonía entre la oferta de programas de formación técnica y la priorización productiva regional de CORFO.

\subsubsection{Demanda por matrículas}

La tabla n. ${ }^{\circ} 11$ presenta la demanda de matrícula en programas de formación técnica que se encuentran dentro de los clusters, para cada una de las regiones. Al respecto, en términos generales, se observa concordancia con la concentración de la oferta en los grandes centros urbanos antes mencionada: para el año 2004, de los 39.871 estudiantes matriculados en programas de formación técnica dentro de algún cluster, 31.297 (78\%) estudiaban en las regiones Metropolitana, Valparaíso y Biobío. Para el año 2009, esta cifra desciende a 72\%.

En cuanto al porcentaje de matrículas en programas de formación técnica que se encuentran dentro de los clusters, la tabla entrega datos que son contrastantes con la oferta. En nueve de las 13 regiones, el porcentaje de matrículas en programas pertenecientes a algún cluster disminuye para el periodo 2004-2009, destacando las

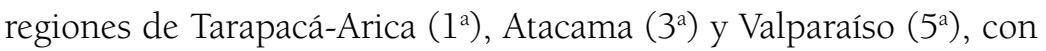
sobre un $14 \%$ de disminución. Asimismo, el promedio de matrículas en programas que tributan a alguno de los clusters para el año 2004 es de un 40\%, y para el 2009, de un 36\%. Estos números no sólo son bajos como indicador, sino que, a su vez, no están correlacionados con la oferta de programas que, para los dos años en estudio, bordea el 50\%. 
172 PERTINENCIA DE LA FORMACIÓN TÉCNICA DE NIVEL SUPERIOR EN RELACIÓN CON LA ESTRATEGIA NACIONAL DE INNOVACIÓN PARA LA COMPETITIVIDAD - Pablo Carrasco, Juan Pablo Venables

Tabla n. ${ }^{\circ} 11$

\begin{tabular}{|c|c|c|c|c|c|c|c|c|}
\hline \multirow[t]{2}{*}{ Región } & \multirow[t]{2}{*}{ Año } & \multicolumn{6}{|c|}{ Cluster } & \multirow{2}{*}{$\begin{array}{c}\% \text { del total } \\
\text { del sistema } \\
\text { representado por } \\
\text { cluster }\end{array}$} \\
\hline & & $\begin{array}{c}\text { Servicios } \\
\text { globales }\end{array}$ & Alimentario & Acuícola & Turismo & Minero & Total cluster & \\
\hline \multirow{2}{*}{$1^{a}$} & 2004 & 815 & 57 & 0 & 355 & 104 & 1.331 & $50 \%$ \\
\hline & 2009 & 614 & 63 & 0 & 302 & 255 & 1.234 & $31 \%$ \\
\hline \multirow{2}{*}{$2^{a}$} & 2004 & 390 & 0 & 0 & 248 & 67 & 705 & $33 \%$ \\
\hline & 2009 & 1.016 & 22 & 0 & 551 & 530 & 2.119 & $37 \%$ \\
\hline \multirow{2}{*}{$3^{a}$} & 2004 & 287 & 52 & 0 & 133 & 58 & 530 & $47 \%$ \\
\hline & 2009 & 443 & 43 & 8 & 98 & 124 & 716 & $33 \%$ \\
\hline \multirow{2}{*}{$4^{a}$} & 2004 & 581 & 152 & 0 & 312 & 0 & 1045 & $34 \%$ \\
\hline & 2009 & 1.238 & 297 & 32 & 312 & 409 & 2.638 & $36 \%$ \\
\hline \multirow{2}{*}{$5^{a}$} & 2004 & 3.186 & 652 & 119 & 1.482 & 31 & 5.470 & $47 \%$ \\
\hline & 2009 & 4.882 & 626 & 73 & 1.456 & 213 & 7.250 & $32 \%$ \\
\hline \multirow{2}{*}{$6^{a}$} & 2004 & 458 & 336 & 0 & 116 & 74 & 984 & $36 \%$ \\
\hline & 2009 & 1.529 & 566 & 0 & 472 & 91 & 2.658 & $35 \%$ \\
\hline \multirow{2}{*}{$7^{a}$} & 2004 & 212 & 228 & 0 & 352 & 0 & 792 & $32 \%$ \\
\hline & 2009 & 1.114 & 767 & 0 & 425 & 56 & 2.362 & $33 \%$ \\
\hline \multirow{2}{*}{$8^{a}$} & 2004 & 1.143 & 252 & 0 & 1.084 & 0 & 2.479 & $29 \%$ \\
\hline & 2009 & 3.425 & 442 & 0 & 1.578 & 95 & 5.540 & $25 \%$ \\
\hline \multirow{2}{*}{$9^{a}$} & 2004 & 743 & 187 & 104 & 363 & 44 & 1.441 & $43 \%$ \\
\hline & 2009 & 1.590 & 235 & 57 & 513 & 107 & 2.502 & $35 \%$ \\
\hline \multirow{2}{*}{$10^{a}$} & 2004 & 361 & 162 & 176 & 425 & 0 & 1.124 & $42 \%$ \\
\hline & 2009 & 1.607 & 645 & 323 & 842 & 0 & 3.417 & $37 \%$ \\
\hline \multirow{2}{*}{$11^{a}$} & 2004 & 55 & 0 & 0 & 35 & 0 & 90 & $27 \%$ \\
\hline & 2009 & 177 & 14 & 54 & 52 & 0 & 297 & $47 \%$ \\
\hline \multirow{2}{*}{$12^{a}$} & 2004 & 251 & 0 & 34 & 222 & 25 & 532 & $43 \%$ \\
\hline & 2009 & 455 & 23 & 6 & 264 & 0 & 748 & $39 \%$ \\
\hline \multirow{2}{*}{ RM } & 2004 & 14.807 & 2.181 & 92 & 5.971 & 297 & 23.348 & $56 \%$ \\
\hline & 2009 & 27.504 & 1.180 & 0 & 7.265 & 236 & 36.185 & $45 \%$ \\
\hline
\end{tabular}

Fuente: elaboración propia a partir de los datos del sistema INDICES del Consejo Nacional de Educación.

Nota: el achurado representa los clusters que son priorizados por cada región.

Coincidentemente con los datos relativos a la oferta, con excepción de las regiones del Maule $\left(7^{\mathrm{a}}\right)$ y Los Lagos-Los Ríos $\left(10^{\mathrm{a}}\right)$ para el año 2004, la mayor cantidad de demanda en las regiones se concentra en el cluster de servicios globales, el cual representa el $58 \%$ de la matrícula total de programas que se encuentran dentro de algún cluster para el año 2004. Esta cifra asciende al 67\% para el 2009. Junto con ello, en las tres regiones donde se prioriza este cluster, se observa un crecimiento importante de la matrícula para el periodo 2004-2009, destacándose la Región del Biobío (8ª), donde esta cifra se triplica. 
En cuanto al cluster alimentario, de aquellas regiones que lo priorizaron dentro de sus estrategias productivas, todas experimentaron un aumento en la matrícula, con excepción de las regiones de Atacama (3a) y Valparaíso ( $\left.5^{a}\right)$, donde se observa una leve disminución de la demanda, lo que coincide con un mantención de la oferta, como se observó en la tabla anterior. Dentro de las regiones con mayor crecimiento destacan la del Maule ( $7^{\mathrm{a}}$ y Los Lagos-Los Ríos $\left(10^{\mathrm{a}}\right)$, cuyo aumento triplica, e incluso casi cuadriplica el número de matrículas para el periodo 2004-2009. En la situación contraria se encuentran las zonas extremas del país, que presentan, en concordancia con su escasa oferta, un crecimiento de matrícula muy precario.

El cluster acuícola, por su parte, presenta una baja matrícula en el nivel país. Con excepción de la Región de Los Lagos-Los Ríos $\left(10^{a}\right)$, que presenta un crecimiento de su matrícula casi al doble para el periodo 2004-2009 -lo cual, no obstante, no se condice con el crecimiento de la oferta, que se quintuplicó en el mismo periodo-, y la Región de Aysén ( $11^{a}$ ), que de no tener estudiantes ni programas de formación técnica relacionados con el sector alimentario ascendió a 54 estudiantes para el 2009, la mayoría de las regiones que priorizaron este cluster presentan un crecimiento de matrícula nulo, o bien decreciente.

De las regiones que priorizaron el cluster turismo de intereses especiales, la mayoría vio aumentar su matrícula de manera importante, con independencia muchas veces del crecimiento de la oferta. Asimismo, de las regiones que señalan el cluster turismo dentro de sus prioridades, la de Valparaíso (5a) y Del Biobío ( $8^{a}$ ) son las que presentan un número mayor de matrículas, pese a que la de Valparaíso (5ª) disminuyó levemente el 2009 en relación con el año 2004, y aun cuando creció en oferta de programas relacionados.

Respecto del cluster minero, en las tres regiones de la zona norte del país se observa un crecimiento importante y armónico con la oferta, destacando la Región de Antofagasta ( $\left.2^{a}\right)$ como la que concentra el mayor número de matrículas para este sector el año 2009, desplazando a la Región de Tarapacá-Arica (1ª), que para el 2004 era la que concentraba el mayor número. 

LA ESTRATEGIA NACIONAL DE INNOVACIÓN PARA LA COMPETITIVIDAD - Pablo Carrasco, Juan Pablo Venables

Tabla n. ${ }^{\circ} 12$

\begin{tabular}{|c|c|c|c|}
\hline Región & Crecimiento en cluster 2004-2009 & Crecimiento vegetativo 2004-2009 & Diferencial \\
\hline $1^{\mathrm{a}}$ & $-7 \%$ & $49 \%$ & -57 \\
\hline $2^{\mathrm{a}}$ & $201 \%$ & $165 \%$ & 35 \\
\hline $3^{\mathrm{a}}$ & $35 \%$ & $94 \%$ & -59 \\
\hline $4^{\mathrm{a}}$ & $152 \%$ & $141 \%$ & 11 \\
\hline $5^{\mathrm{a}}$ & $33 \%$ & $93 \%$ & -60 \\
\hline $6^{\mathrm{a}}$ & $170 \%$ & $179 \%$ & -9 \\
\hline $7^{\mathrm{a}}$ & $198 \%$ & $183 \%$ & 15 \\
\hline $8^{\mathrm{a}}$ & $123 \%$ & $160 \%$ & -37 \\
\hline $9^{\mathrm{a}}$ & $74 \%$ & $112 \%$ & -38 \\
\hline $10^{\mathrm{a}}$ & $204 \%$ & $248 \%$ & -44 \\
\hline $11^{\mathrm{a}}$ & $230 \%$ & $94 \%$ & 136 \\
\hline $12^{\mathrm{a}}$ & $41 \%$ & $55 \%$ & -15 \\
\hline $\mathrm{RM}$ & $55 \%$ & $92 \%$ & -37 \\
\hline
\end{tabular}

Fuente: elaboración propia a partir de los datos del sistema INDICES del Consejo Nacional de Educación.

Relacionando el crecimiento de la demanda por programas que se encuentran clasificados en algún cluster con el crecimiento vegetativo del total de programas de formación técnica, se observa que las mismas seis regiones que presentaban un crecimiento negativo de la oferta también decrecen en cuanto a la matrícula; éstas son: Tarapacá-Arica $\left(1^{a}\right)$, Atacama ( $\left.3^{a}\right)$, Valparaíso (5a), O’Higgins (6a), La Araucanía (9a), y la Región Metropolitana (RM). Ahora bien, además de estas regiones, otras tres presentan un crecimiento negativo de la demanda, pese a que su oferta se mantuvo o creció levemente: las regiones del Biobío $\left(8^{a}\right)$, Los Lagos-Los Ríos $\left(10^{a}\right)$ y Magallanes $\left(12^{a}\right)$. A diferencia de lo anterior, la Región de Aysén (11 ${ }^{a}$ ) presenta un crecimiento de los programas de formación técnica relacionados con los cluster de 136\% por sobre el crecimiento vegetativo de la región.

\section{Conclusiones}

Si bien distintos actores vinculados al sistema educacional y de innovación en Chile han señalado la importancia de generar una articulación entre la formación técnica de nivel superior y la Estrategia Nacional de Innovación para la Competitividad, transcurridos cinco años de su creación, no existe evidencia que permita afirmar que esto se haya producido ni que actualmente se encuentre en desarrollo. 
Para que el país pueda alcanzar mayores niveles de desarrollo en el mediano y largo plazo, las estrategias en capital humano deberían avanzar en varios frentes, dentro de los cuales se destacan: 1) establecer un sistema de aprendizaje a lo largo de toda la vida, 2) asegurar la conexión entre los distintos canales de formación superior, 3) desarrollar un sistema de calificaciones basado en competencias laborales para la formación técnica y la capacitación, y 4) articular los distintos niveles de formación técnica y capacitación con los requerimientos del mercado laboral (CNIC, 2007). Sin embargo, hoy, la articulación entre los distintos niveles de formación -institucional y curricular- es débil, así como también la vinculación del sistema educacional con las necesidades del mercado (Kis y Field, 2009).

Pese a que parece existir consenso respecto de hacia dónde debe avanzarse en materia de formación técnica de nivel superior, los resultados no exhiben progresos en este sentido. Aun cuando el crecimiento experimentado durante el periodo 2004-2009 por los programas de formación técnica de nivel superior fue mayor -al menos en cuanto al crecimiento de las matrículas- que el crecimiento del sistema de educación superior en su conjunto (53\% de crecimiento en la oferta y $112 \%$ en demanda, en contraposición a un $57 \%$ en oferta y un $45 \%$ en demanda para todo el sistema), el crecimiento de los programas de formación técnica que se encuentran dentro de los clusters productivos identificados por la Estrategia Nacional de Innovación para la Competitividad no ha seguido la misma dirección.

Como se observa en el cuerpo del estudio, al comparar el crecimiento de la oferta y la demanda de los clusters en relación con el crecimiento vegetativo del total de programas de formación técnica, el crecimiento de la oferta de los clusters es $-13 \%$. Todos presentan un crecimiento negativo o nulo, con excepción del cluster acuícola.

Más aun, articulando estos datos con los de demanda, se pudo observar que no existe consistencia ni coherencia entre ambos. Mientras la oferta de programas dentro de los clusters presenta un crecimiento negativo de $13 \%$, la demanda mermó en un $45 \%$. Junto con ello, el cluster acuícola -único cluster que presenta un crecimiento positivo en 
la oferta en relación con el vegetativo- es, a su vez, aquel que presenta un menor crecimiento de matrícula, alcanzando un $-107 \%$.

Asimismo, el cluster de servicios globales sólo es priorizado por las regiones Metropolitana, de Valparaíso y Concepción, lo que resulta coherente con un sector productivo propio de las grandes urbes. Sin embargo, todas las regiones, independientemente de que no priorizaran este sector dentro de sus estrategias de desarrollo, concentran la mayor parte de su oferta y demanda en este cluster, alcanzando, en el nivel nacional, más de dos tercios de la oferta de programas de formación técnica clasificados dentro de algún cluster. En esta misma línea, sectores que requieren mayor especialización y en los cuales Chile posee ventajas competitivas de recursos naturales por sobre otros países, como la minería y la acuicultura, presentan un crecimiento de su oferta muy precario.

Por otro lado, se pudo evidenciar que un $67 \%$ del total de los programas de formación técnica pertenecientes a los clusters son dictados por instituciones acreditadas. Bajo el entendido que la acreditación institucional representa un sello de calidad de la gestión académica y administrativa de las instituciones de educación superior, cabe preguntarse por el grado de relación que existe entre esta certificación y la respuesta que las instituciones entregan a las necesidades del mercado laboral.

Respecto del cluster minero, si bien presenta un crecimiento en la oferta y demanda de sus programas de formación técnica bajo en relación con las necesidades del país, es el único que consiguió un crecimiento de demanda positivo, alcanzando un $90 \%$ por sobre el crecimiento vegetativo. Junto con ello, su aumento se concentra principalmente en el extremo norte del país, específicamente en la Región de Antofagasta, lo que muestra la coherencia de su crecimiento.

En conclusión, los datos demuestran de manera fehaciente la necesidad de generar una mayor articulación entre la oferta y demanda de la formación técnica de nivel superior con las necesidades productivas del país. 
Chile requiere de más y mejores técnicos, pero también de una oferta y demanda alineadas con el desarrollo productivo que pretende alcanzar. En este sentido, la pertinencia de la formación técnica, entendida como atributo de calidad del sistema, necesita de políticas públicas que orienten la oferta. Así, urge avanzar en el fortalecimiento del sector, atendiendo las evidentes diferencias de tamaño, recursos y capacidades que existen entre los distintos actores institucionales, y considerando, asimismo, que sólo los centros de formación técnica concentran el 55\% de la oferta adscrita a los clusters, identificados, lo que los vuelve especialmente atractivos de ser beneficiarios de una política pública de fortalecimiento mayormente activa.

Pero también se requiere de una acción más decidida en materia de provisión de información pública asequible para el postulante, que le permita adoptar decisiones informadas respecto de las distintas opciones dentro del sistema de educación superior. Materias como empleabilidad proyectada, requerimientos del mercado laboral y orientaciones de política pública al respecto, deben ser variables especialmente consideradas en un sistema de información cuyo foco esté en orientar y promover la formación para el trabajo, favoreciendo así la consecución de las metas de desarrollo productivo definidas por la Estrategia Nacional de Innovación para la Competitividad.

\section{Referencias bibliográficas}

AUR (2009) Revista Chilena de Estudios Regionales n. ${ }^{\circ}$ 1. Agrupación de Universidades Regionales y Red Sinergia Regional. Julio 2009.

Bitrán, E. (2009) La Estrategia Nacional de Innovación para la Competitividad. Serie Seminarios Internacionales (12), pp. 55-68. Política de Educación Superior: Explorando Horizontes, Riesgos y Posibilidades.

Brunner,J.J. (2006) Mercados universitarios: Ideas, instrumentaciones y seis tesis en conclusión. Disponible en: http://mt.educarchile.cl/MT/jjbrunner/ archives/libros/MERC_UNI_DEF/LA\%20UNIVERSIDAD\%20 FRENTE\%20AL\%20MERCADO_DEFhtml

CNA (2010) Términos de referencia para las áreas contempladas en el proceso de acreditación institucional. Disponible en: http://www.cnachile.cl/ docs/materiales/anexo2-term-ref-ai.pdf 
CNED (2005) Estadísticas y Bases de Datos sistema INDICES. Consejo Nacional de Educación. Disponible en: http://www.cned.cl

CNED (2010) Estadísticas y Bases de Datos sistema INDICES. Consejo Nacional de Educación. Disponible en: http://www.cned.cl

CNIC (2007) Hacia una Estrategia de Innovación para la Competitividad. Volumen I. Informe del Consejo Nacional de Innovación para la Competitividad. Chile.

CNIC (2008) Hacia una Estrategia de Innovación para la Competitividad. Volumen II. Informe del Consejo Nacional de Innovación para la Competitividad. Chile.

CONICYT (2010) Sistema Bicentenario Becas Chile. Becas de Perfeccionamiento Técnico. Disponible en: http://www.becaschile.cl/becas/detalle/ especializacion/tecnico/

CORFO (2010) Programa Nacional de Clusters. Disponible en: http://www. corfo.cl/clusters

Kis, V.; Field, S. (2009) Learning for Jobs. OECD Reviews of Vocational Education and Training. Chile: a first report. Organization for Economic Cooperation and Development (OECD).

MINEDUC (2009) Bases para una Política de Formación Técnico-Profesional en Chile. Informe Ejecutivo. Santiago de Chile: Ministerio de Educación.

MINEDUC (2010) Sistema Nacional de Información de la Educación Superior (SIES). Ministerio de Educación. Chile. Informe sobre Retención de Primer Año de las Carreras. Cohorte 2007. Disponible en: http://www. divesup.cl/sies/?page_id=36

SERNAGEOMIN (2010) Recursos Minerales Metálicos. Servicio Nacional de Geología y Minería. Disponible en: http://www.sernageomin.cl/index. php?option=com_content $\&$ task $=$ view\&id $=129 \&$ Itemid $=186$

UFRO (2008) Estudio base de capacidades instaladas en regiones en materia de ciencia, tecnología e innovación. Instituto de Desarrollo Local y Regional (IDER), Universidad de La Frontera. Disponible en: http:// www.ider.cl

Recibido: 01/09/2010

Aceptado: 02/11/2010 


\section{Anexo 1: Carreras por cluster año 2004 y $2009^{9}$}

\section{Año 2004}

\begin{tabular}{|c|}
\hline Acuicultura \\
\hline Acuicultura; producción acuícola; recursos acuáticos. \\
\hline Alimentario \\
\hline $\begin{array}{l}\text { Agrícola; Agrícola y ganadero; Agroindustrial; Agrónomo; Administración agropecuaria; } \\
\text { Agroindustria; Agroindustria m/productos cárnicos; Agronomía; Alimentos; Conservación de } \\
\text { alimentos por frío; Control de alimentos; Control de calidad agrícola y agroindustrial; Gestión } \\
\text { de negocios agrícolas; Industria de alimentos; Microbiología industrial de alimentos; Producción } \\
\text { agropecuaria; Producción de alimentos; Tecnología agrícola; Tecnología agrícola m/cultivos y } \\
\text { ganadería; Tecnología agrícola m/cultivos, frutales y vides; Tecnología de procesos agroindustriales; } \\
\text { Tecnología administración m/agronegocios; Tecnología industrial de alimentos; Tecnología } \\
\text { industrial de los alimentos; Tecnología industrial de los alimentos del mar; Vitivinicultura; Experto } \\
\text { agrario; Hortícola; Pecuario. }\end{array}$ \\
\hline Minería \\
\hline $\begin{array}{l}\text { Plantas metalúrgicas; Procesamiento de minerales; Tecnología minería y metalurgia; Topografía; } \\
\text { Topógrafo. }\end{array}$ \\
\hline Servicios globales \\
\hline $\begin{array}{l}\text { Analista de Sistemas; Analista Financiero; Analista Programador; Analista Programador } \\
\text { Computacional; Asistente Gerencial y Comercial; Auditor; Administración Bancaria y Comercial; } \\
\text { Administración de Empresas Especialista en Comercio Exterior; Administración de Empresas } \\
\text { especialista Proyectos y Finanzas; Administración de Empresas m/Comercialización y Personal; } \\
\text { Administración de Empresas m/Computación; Administración de Empresas m/Contabilidad } \\
\text { y Tributación; Administración de Empresas m/Finanzas; Administración de Empresas m/ } \\
\text { Finanzas, Comerc., Personal; Administración de Empresas m/Gestión; Administración de } \\
\text { Empresas m/Gestión de Locales Comerciales; Administración de Empresas m/Gestión y } \\
\text { Evaluación Proyectos PYME; Administración de Empresas m/Logística; Administración de } \\
\text { Empresas m/Marketing; Administración de Empresas m/Marketing y Gestión Comercial; } \\
\text { Administración de Empresas m/Marketing y Ventas; Administración de Empresas m/ } \\
\text { Personal; Administración de Empresas m/Personal, Finanzas; Administración de Empresas } \\
\text { m/Recursos Humanos; Administración de Sistemas Logísticos; Administración Comercio } \\
\text { Exterior; Administración Gestión de Comercio Exterior; Administración; Administración m/ } \\
\text { Op. Financieras; Administración Comercial; Administración de Empresas; Administración } \\
\text { de Empresas; Administración de Empresas m/Marketing tec. Nivel Sup. Administración de } \\
\text { Operaciones; Administración de Operaciones; Administración de Procesos Productivos; } \\
\text { Administración de Redes Computacionales; Administración de Redes y Soporte Computacional; } \\
\text { Administración de Redes y Soportes; Administración de Sistemas Logísticos; Administración } \\
\text { Financiera; Administración Financiera y Comercial; Administración m/ Finanzas - Marketing; }\end{array}$ \\
\hline
\end{tabular}

9 Todas las carreras reciben la denominación de Técnico de Nivel Superior, la cual antecede al nombre que se señala, pero por razones de espacio no se repite para cada una. 
Administración m/Gestión de Empresas; Administración m/Marketing y Comercialización; Administración m/Marketing y Ventas; Administración y Gestión de Empresas; Administración y Soporte de Redes; Administración y Soporte Tecnológ. Proc. Produc.; Análisis de Sistemas de Inf. Administrativa; Análisis y Programación de Sist. Comp.; Auditoría; Comercio Electrónico; Comercio Exterior; Comercio Exterior; Comercio Exterior m/Gestión Aduanera; Comercio Exterior m/Transporte Internacional; Comercio Internacional; Comercio Internacional m/tratados de libre comercio; Computación; Computación e Informática; Computación y Tecnología de la Información; Comunicación Multimedia; Comunicación y Relaciones Públicas; Conectividad y Redes; Construc. y Administración de Redes Analógicas y Digitales tec. Nivel Sup; Contabilidad; Contabilidad Computacional; Contabilidad General; Contabilidad Gral. Computacional; Contabilidad Gral. m/Computación; Contabilidad Gral. m/Informática; Contabilidad Gral. m/Legislación Tributaria; Contabilidad y Auditoría; Desarrollo de Sistemas Computacional estec. Nivel Sup.; Diseño Gráfico Multimediatec. Nivel Sup.; Diseño Gráfico y Multimedia; Gestión Comercial; Gestión y Soporte de Redes; Gráfica Editorial y Digital; Higiene Ambiental; Ilustración Digital; Informática; Informática m/Análisis y Prog. de Sistemas; Informática m/ Análisis y Programación; Informática m/Redes y Sistemas; Informática m/Redes y Sistemas de comunicaciones; Logística; Logística Operativa; Marketing; Negocios Internacionales; Producción de Multimedia; Producción de Sistemas e Internet; Producción de Sistemas Multimedia e Internet; Producción Gráfica Digital; Producción Multimedial; Programación Computacional; Programación de Aplicaciones Computacionales; Programación de Computadores; Programación Computación; Programación Computación e Informática; Programación Webmaster; Programación y Análisis de Sistemas; Redes de Computadoras; Redes y Comunicaciones; Redes y Conectividad Digital; Secret. Bilingüe de Gestión Empresarial m/; Secretariado Bilingüe de Gestión Empresarial; Secretariado Ejec. Bilingüe m/Comercio Exterior; Sistemas Informáticos; Tecnología Informática m/; Telecomunicaciones; Telecomunicaciones y Telemática; Telecomunicaciones (C/Módulos) Téc. Nivel Sup.; Telecomunicaciones y Telemáticatec. Nivel Sup.; Telemática y Telecomunicaciones; Ventas y Negociación Comercial; Financiero; Interpretación de Lace Inglés-Castellano; Programación Computacional; Programación Computación; Programación Computación e Informática; Programador Analista; Programador de Aplicaciones Computacionales; Secretariado Ejec. Bilingüe m/; Secretariado Ejec. Computacional; Secretariado Ejec. Computación; Secretariado Ejec. m/ Op.Computadores; Secretariado Ejecutivo Bilingüe; Traducción Inglés-Castellano asistida por Computación; Traducción Inglés-Español; Traductor Inglés-Español; Traductor Inglés-Español m/; Webmaster; Webmastertecnólogo Telecomunicaciones.

\begin{tabular}{l} 
Turismo \\
\hline Adm. de negocios gastronómicos; Adm. de recursos turísticos m/ecoturismo; Adm. de servicios \\
turísticos m/; Adm. hotelera m/; Administración hotelera; Cocina internacional; Cocina \\
internacional y trad. chilena; Dirección y producción de eventos; Gastronomía; Gastronomía \\
internacional; Gastronomía internacional m/eventos; Gastronomía y cocina internacional; \\
Gastronomía y pastelería internacional; Hotelería internacional; Ilustración; Marketing de negocios \\
hoteleros; Organización y producción de eventos; Produc. gastronómica m/; Producción y gestión \\
de eventos; Relaciones públicas; Relaciones públicas y producción de eventos; Servicios hoteleros; \\
Servicios turísticos m/turismo aventura; Servicios turísticos m/viajes y turismo; Téc. gestión de \\
proyectos turísticos; Turismo; Turismo m/adm. de recursos turísticos; Turismo m/ecoturismo; \\
Turismo m/empresas de viajes; Turismo m/tráfico y carga aérea; Turismo y hotelería.
\end{tabular}




\section{Año 2009}

Acuicultura

Acuicultura; Acuicultura y Medio Ambiente; Buceo Comercial; Pesca Artesanal; Producción Acuícola; Producción de Peces; Recursos Marinos; Salmonicultura; Salud de Peces; Tecnología en Acuicultura.

Alimentario

Agrícola; Agrícola y Ganadero; Agroindustrial; Agrónomo; Agropecuario; Administración y Producción Agropecuaria y Agroindustrial; Administración Agropecuaria; Agricultura Ecológica; Agroindustria; Agronegocios; Agronomía; Alimentos; Biotecnología Industrial; Control Agroindustrial; Frutales; Gestión Agropecuaria; Gestión de Predios Agrícolas; Industria Alimentaria; Industrias Agroalimentarias; Medicina Veterinaria; Tecnología Agrícola; Tecnología en Vitivinicultura; Tecnología Industrial de los Alimentos; Tecnología Pecuaria; Tecnología y Administración Agrícola; Tecnología y Administración Pecuaria; Veterinaria; Vinicultura; Viticultura y Enología; Vitivinicultura; Experto Agrario; Hortícola; Pecuario; Producción Frutícola Intensiva y Sustentable; Producción Hortícola Intensiva y Sustentable; Veterinario.

Minería

Eléctrico Instrumentista Mantenedor Plantas Mineras; Explotación de Minas; Geología; en Geominería; Maquinaria Pesada; Metalurgia; Minas; Operaciones en Minas; Operaciones Mineras; Tecnología en Metalurgia; Tecnología en Operaciones Mineras; Topografía; Laboratorista Químico Minero; Mecánico Mantenedor Plantas Mineras; Tecnólogo en Metalurgia.

Servicios globales

Administrador Financiero; Analista de Sistemas; Analista Financiero; Analista Programador; Analista Programador Computacional; Asistente Bilingüe de Gestión Empresarial; Asistente Ejecutivo Bilingüe; Asistente Ejecutivo Computacional; Asistente Gerencial y Comercial; Asistente Gestión Ejecutiva Bilingüe y Computacional; Contabilidad; Contador General; Administración; Administración Bancaria; Administración Bancaria y Financiera; Administración Bancaria y Técnicas Contables; Administración Comercial; Administración Contable y Financiera; Administración de Empresas; Administración de Empresas Bancaria y Financiera; Administración de Logística; Administración de Redes Computacionales; Administración de Redes y Soporte Computacional; Administración de Sistemas Logísticos; Administración de Ventas; Administración en Comercio Exterior; Administración Financiera; Administración Financiera y Comercial; Administración y Gestión de Empresas; Administración y Gestión Empresarial; Administración y Soporte de Redes; Administración y Soporte de Redes Computacionales; Análisis Computacional de Sistemas; Análisis de Sistemas; Análisis de Sistemas Computacionales; Animación Digital; Aplicaciones Computacionales; Auditoría; Auditoría Computacional; Comercio Electrónico; Comercio Exterior; Comercio Exterior y Agenciamiento Aduanero; Comercio Internacional; Comercio Mayorista y Exterior; Comercio Naviero; Comercio y Transporte Internacional Bilingüe; Computación; Computación e Informática; Computación y Tecnologías de la Información; Comunicación Digital y Gestión de Medios en la Web; Comunicación Multimedia; Conectividad y Redes; Construc. y Administración de Redes Analógicas y Digitales; Contabilidad; Contabilidad Computacional; Contabilidad General; Computacional; Contabilidad y Auditoría; Contabilildad; Control de Alimentos; Diseño Digital; Diseño Gráfico Multimedial; Diseño Gráfico y Multimedia; Diseño y Creación de Sitios Web; Diseño y Producción Gráfica Digital; Diseño y Programación de Videojuegos; Diseño y Programación Multimedia; Electrónica Computacional; Finanzas; Gestión Comercial; Gestión Contable y Tributaria; Gestión de Empresas; Gestión de Negocios; Gestión de Servicios Financieros; Gestión Empresarial; Gestión Financiera; Gestión Logística; Gestión y Soporte de Redes; Gráfica Editorial y Digital; Ilustración Digital; Informática; Informática y Redes; 
Informática y Seguridad; Inglés Comunicacional; Interpretariado Inglés Alemán; Interpretariado Inglés Castellano; Logística; Logística Empresarial; Logística Operativa; Marketing y Gestión Comercial; Plataformas Informáticas; Producción de Multimedia; Producción Gráfica Digital; Producción Gráfica Multimedia; Producción Informática Multimedia; Producción Multimedial y Diseño Web; Programación Computacional; Programación Computacional; Programación de Aplicaciones Computacionales; Programación de Microcomputadores; Programación en Computación; Programación en Computación e Informática; Programación Webmaster; Programación y Análisis de Sistemas; Programación y Diseño Informático; Publicidad y Marketing; Redes Computacionales; Redes de Computadores; Redes y Telecomunicaciones; Relaciones Públicas de Negocios; Secretariado Ejecutivo Bilingüe; Soporte Computacional; Soporte y Mantención de Redes; Soporte, Conectividad y Redes; Tecnología en Producción Multimedia y Diseño Web; Tecnología Informática; Telecomunicaciones y Telemática; Telecomunicaciones, Conectividad y Redes; Traducción e Interpretación Inglés Español; Traducción e Intérprete de Contacto; Traducción e Intérprete Inglés Español; Traducción Inglés Español; Traducción Inglés Español; Sistemas Digitales; Ventas y Marketing; Ventas y Negociación Comercial; Financiero; Interpretación de Enlace Inglés Castellano; Intérprete en Negocios Inglés Español; Intérprete Inglés Español; Operaciones Logísticas; Programador Computacional; Programador de Aplicaciones Computacionales Secretariado Bilingüe; Secretariado Ejecutivo Bilingüe; Secretariado Ejecutivo Computacional; Secretariado Ejecutivo en Computación; Secretariado Ejecutivo Traductor Bilingüe; Secretariado Gerencial Bilingüe; Soporte en Redes; Tecnología en Administración de Empresas; Tecnología en Informática; Tecnólogo en Informática; Traducción Inglés Español; Traductor Bilingüe Inglés Español

\begin{tabular}{l}
\hline Turismo \\
\hline Administración de Recursos Turísticos; Administración de Negocios Gastronómicos; Administración \\
Hotelera; Alimentación Colectiva; Cocina Internacional y Tradicional Chilena; Cocina Nacional \\
e Internacional; Comunicación y Relaciones Públicas; Dirección y Producción de Eventos; \\
Ecoturismo; Gastronomía; Gastronomía Internacional; Gastronomía y Cocina Internacional; \\
Gastronomía y Pastelería Internacional; Gestión de Eventos y Producción Cultural; Gestión de \\
Negocios Gastronómicos; Gestión y Producción de Eventos; Guía de Turismo; Hotelería; Hotelería \\
Marítima Bilingüe; Hotelería y Turismo; Hoteles y Restaurantes; Marketing de Negocios Hoteleros; \\
Organización y Producción de Eventos; Producción Gastronómica; Producción y Gestión de \\
Eventos; Relaciones Públicas; Relaciones Públicas y Producción de Eventos; Relaciones Públicas \\
y Publicidad; Salud y Terapias Naturales; Servicios Gastronómicos Hoteleros y de Restaurante; \\
Servicios Turísticos; Tecnología en Dirección y Producción de Eventos; Tecnología en Gastronomía; \\
Terapias Naturales; Turismo; Turismo Aventura; Turismo Bilingüe; Turismo y Hotelería; Guía de \\
Turismo.
\end{tabular}

